\title{
Gene expression profiling of rubella virus infected primary endothelial cells of fetal and adult origin
}

\author{
Henriette Geyer ${ }^{1 *}$, Michael Bauer ${ }^{2}$, Jennifer Neumann ${ }^{3}$, Amy Lüdde$^{1}$, Paul Rennert ${ }^{1}$, Nicole Friedrich', \\ Claudia Claus ${ }^{4}$, Ludmilla Perelygina ${ }^{5}$ and Annette Mankertz ${ }^{1}$
}

\begin{abstract}
Background: Rubella virus (RV) infection is usually a mild illness in children and adults. However, maternal infection during the first trimester of pregnancy can lead to congenital rubella syndrome (CRS) in the infant. Fetuses with CRS show damage to the endothelium of the heart and blood vessels; thus, it has been speculated that the clinical manifestations associated with CRS may be a result of endothelial cells persistently infected with RV. Here, we compared the effects of RV infection on gene expression in primary endothelial cells of fetal (HUVEC) and of adult (HSaVEC) origin by transcriptional profiling.

Results: More than $75 \%$ of the genes differentially regulated following RV infection were identical in both cell types. Gene Ontology (GO) analysis of these commonly regulated genes showed an enrichment of terms involved in cytokine production and cytokine regulation. Increased accumulation of inflammatory cytokines following RV infection was verified by protein microarray. Interestingly, the chemokine CCL14, which is implicated in supporting embryo implantation at the fetal-maternal interface, was down-regulated following RV infection only in HUVEC. Most noticeably, when analyzing the uniquely regulated transcripts for each cell type, GO term-based cluster analysis of the down-regulated genes of HUVEC revealed an enrichment of the GO terms "sensory organ development", "ear development" and "eye development".

Conclusion: Since impairment in vision and hearing are the most prominent clinical manifestations observed in CRS patients, the here detected down-regulated genes involved in the development of sensory organs sheds light on the molecular mechanisms that may contribute to the teratogenic effect of RV.
\end{abstract}

Keywords: Rubella virus, Congenital rubella syndrome, Gene expression, Sensory organ development, Teratogenicity, Endothelial cells

\section{Background}

Rubella virus (RV) is a single-stranded RNA virus of positive polarity and belongs to the family Togaviridae. RV infection typically causes mild symptoms such as rash and fever in children and adults; however, its teratogenicity is still a public health concern. Maternal infection early in pregnancy can lead to a combination of birth defects in infants, collectively called congenital rubella syndrome (CRS) $[1,2]$. Transplacental transmission of the virus is high in the first weeks of gestation [3], and if infection

\footnotetext{
*Correspondence: geyerh@rki.de

'Division 12, "Measles, Mumps, Rubella, and Viruses Affecting

Immunocompromised Patients", Robert Koch Institute, 13353 Berlin, Germany Full list of author information is available at the end of the article
}

occurs, the virus is able to infect almost any organ, thereby establishing a chronic infection in the developing fetus [4]. In RV-infected embryos and fetuses, vascular abnormalities, such as lesions in the endothelium of the heart and blood vessels, have been described as the most frequently observed pathological findings [5-8]. Common clinical manifestations associated with CRS are patent ductus arteriosus, pulmonary artery stenosis and valvular stenosis [9]. Other frequently observed defects include cataracts, glaucoma, sensorineural deafness and psychomotor retardation [10]. Despite the introduction of an effective vaccine and global vaccination programs, approximately 110,000 CRS cases are estimated to still occur worldwide per year [11]. 
RV infection during the critical stages of organ development and in a setting where a mature immune system is absent is believed to account for the clinical manifestations of CRS. However, the exact molecular mechanisms by which RV causes CRS are still poorly understood. Previous work has shown that RV interferes with cellular proliferative pathways, alters cytoskeletal structures, and induces mitochondrial changes, leading to the hypothesis that virus-induced apoptosis contributes to the teratogenic effects of RV (reviewed in J. Y. Lee and D. S. Bowden [12] and D. M. Knipe and P. M. Howley [13]). However, most of these findings are derived from studies using immortalized adult cell lines (e.g. Vero, A549, and BHK-21 cells). These cell lines differ considerably from fetal cells in vivo in terms of gene expression, metabolism, and growth rate, and therefore give only limited insight into the effects of RV infection during embryonic development.

Microarray gene expression analysis provides a valuable tool to comprehensively examine and identify pathways that are affected by virus infection. Previous transcriptional profiling analysis following RV infection of primary human fibroblasts derived from a whole embryo [14], as well as the ECV304 cell line which exhibit both endothelial and epithelial characteristics [15], revealed that RV induces a robust interferon-stimulated gene response. However, since endothelial cells are believed to play a major role in RV-induced teratogenesis, our studies focused on the gene expression changes of an infection caused by a wild type RV isolate (Wuerzburg-12) in primary fetal endothelial cells derived from the human umbilical vein (HUVEC) and adult endothelial cells derived from the human saphenous vein (HSaVEC). By comparing up- and down-regulated genes in the endothelial cells of fetal and adult origin using Gene ontology (GO) term analysis, we were able to identify differences in biological processes and pathways between HUVEC and HSaVEC. We believe that these differences in gene expression after infection of endothelial cells of adult and fetal origin provide new insights into the molecular mechanisms involved in RV-induced teratogenicity.

\section{Results}

\section{Primary endothelial cells are permissive for RV}

$\mathrm{RV}$ is characterized by a relatively slow replication cycle $[16,17]$ and has been shown to infect different cell types asynchronously, even at a high MOI $[14,18]$. However, L. Perelygina, et al. [19] found that RV infection at an MOI $\geq 10$ produced synchronously infected HUVEC cultures. To determine the optimal time point for gene expression analyses and in order to see if HSaVEC are also synchronously infected, viral replication was analyzed by growth curves and viral capsid protein levels were determined by flow cytometry. The immortalized cell lines Vero76 and A549 have been used successfully for producing high RV titers [13] and were therefore used as controls in the following experiments. Growth kinetics showed that RV effectively replicates in the monolayer of fetal and adult primary endothelial cells. Comparable titers were observed after infection of HUVEC, A549, and Vero76; the infection of HSaVEC produced slightly lower viral titers (Fig. 1(a)). However, the viral titer remained almost constant during the observation period of five days for all cell types. Since it has been shown that the efficiency of RV egress is strongly dependent on the cell line [20], we quantified the titers of both extracellular and cell-associated virus, revealing that a similar amount of extracellular and cellassociated virus was produced in Vero76 cells. In contrast, the amount of extracellular virus was approximately one log higher in the supernatant than cellassociated in HUVEC, HSaVEC, and A549 cells. This is in accord with previous studies and suggests a more efficient viral egress in human cell lines [19]. Only moderate cytopathic effects (CPE), such as cell rounding and detachment, were detectable for all cell types two days post infection (dpi) (Fig. 1 (b)).

In contrast to L. Perelygina, et al. [19] we did not detect synchronous infection in HUVEC by flow cytometry (Fig. 1 (c)). Approximately one fourth of the primary endothelial cells were RV capsid positive $23 \mathrm{~h}$ post infection (hpi). Capsid protein levels increased over time, reaching about $50 \%$ of capsid positive cells in the primary endothelial cells 48 hpi. Interestingly, immortalized cell lines Vero76 and A549 cells expressed more viral capsid than HUVEC and HSaVEC, indicating that RV is able to infect these cell lines more efficiently.

\section{Similar changes in gene expression pattern after RV infection of fetal and adult endothelial cells}

The viral life cycle of RV is completed approximately 24 hpi $[16,19]$. Due to asynchronous infection of the primary endothelial cells, and in order to have a relatively high number of infected primary endothelial cells while still avoiding multiple viral life cycles, 36 hpi was chosen for analysis of gene expression. HUVEC and HSaVEC were infected in triplicate with the clinical isolate $\mathrm{Wb}-12$ at an MOI of 10. Non-infected cells served as the control. Total RNA was harvested 36 hpi and was used for microarray analysis of host cell transcription. To compare the gene expression of infected and non-infected cells, ratios were calculated by using normalized signal intensities of infected-and non-infected cells. Genes that exhibited a fold change in gene expression $\geq 4$ and $\leq-4$ with an ANOVA p-value of $\leq 0.01$ were chosen for further analysis (Additional file 1).

Gene expression data of virus-infected to non-infected cells were compared to each other to determine the 


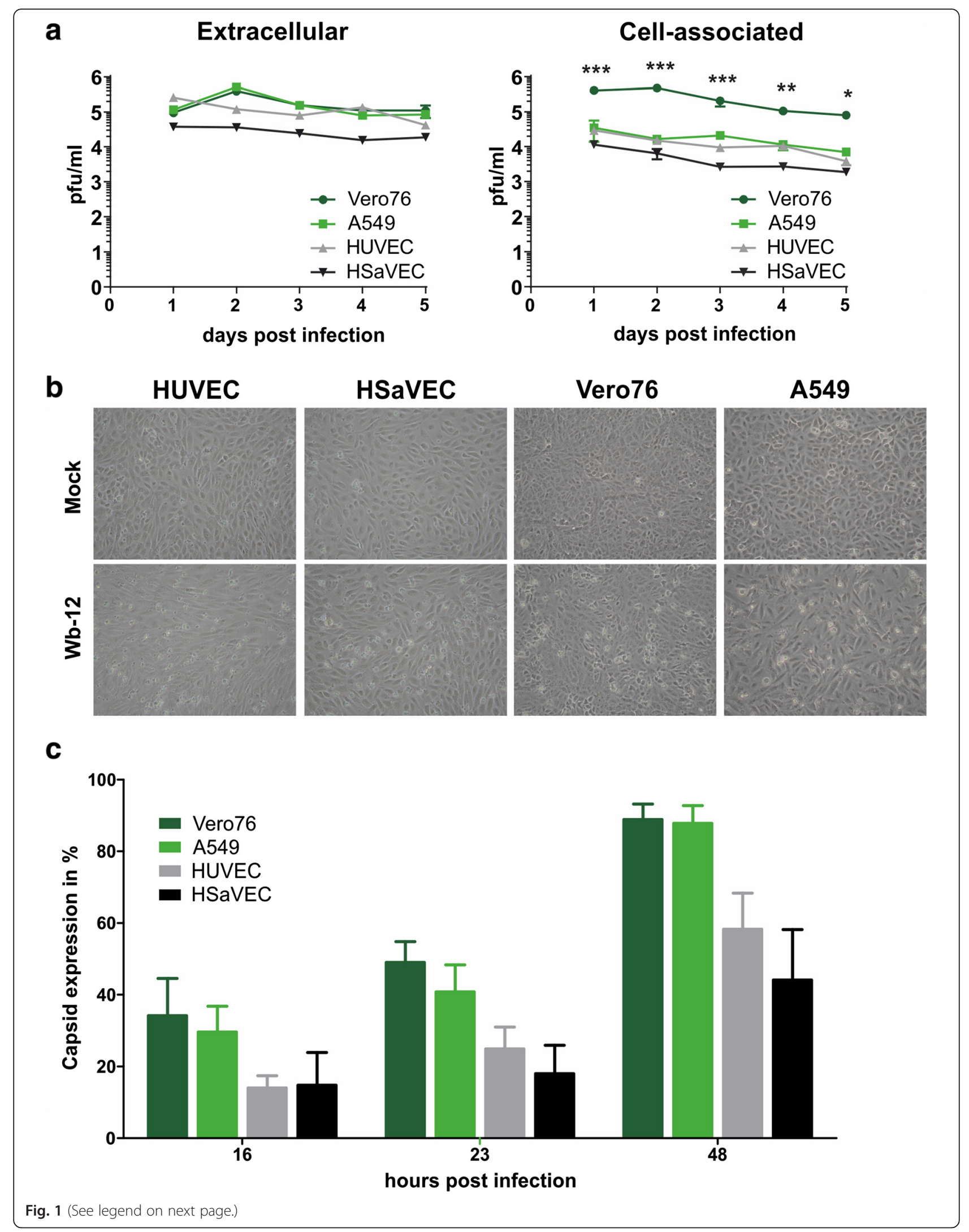


(See figure on previous page.)

Fig. 1 RV replication in primary endothelial cells and RV-permissive cell lines. a RV replication kinetics in HUVEC, HSaVEC, Vero76, and A549 cells. Cells were infected with RV at an $\mathrm{MOI}$ of 5 . At the indicated time points, cell culture supernatants or cell lysates were titered in duplicate on Vero76 cells. The data are represented as the mean of two independent experiments \pm standard deviation (SD). The data were analyzed by twoway ANOVA with the Bonferroni correction for multiple comparisons $\left({ }^{*}, p \leq 0.05 ;{ }^{* *}, p \leq 0.01 ;{ }^{* *}, p \leq 0.001\right)$. b Phase contrast pictures of non-infected cells or cells infected with RV with an MOI of 10 two dpi. c Capsid protein levels of RV-infected cell types at various time points post infection. Cells were infected with an $\mathrm{MOl}$ of 5 and harvested at the indicated time points and analyzed by flow cytometry. Intracellular staining was carried out using a capsid-specific antibody and anti-mouse-Cy5 serum as a secondary antibody. Non-infected cells served as the control. All experiments were performed three times. Error bars represent means \pm SD

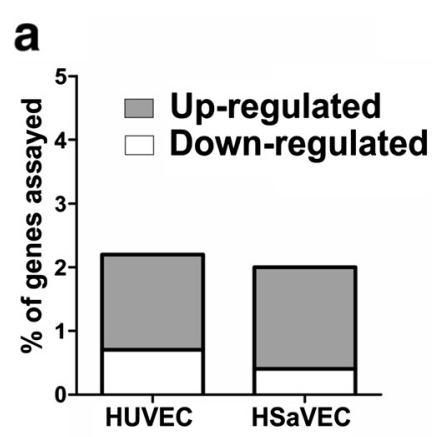

b

\section{HUVEC}

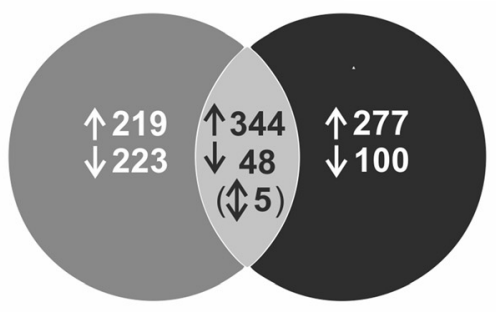

个Up-regulated

$\downarrow$ Down-regulated

$\Uparrow$ Contra-regulated

C

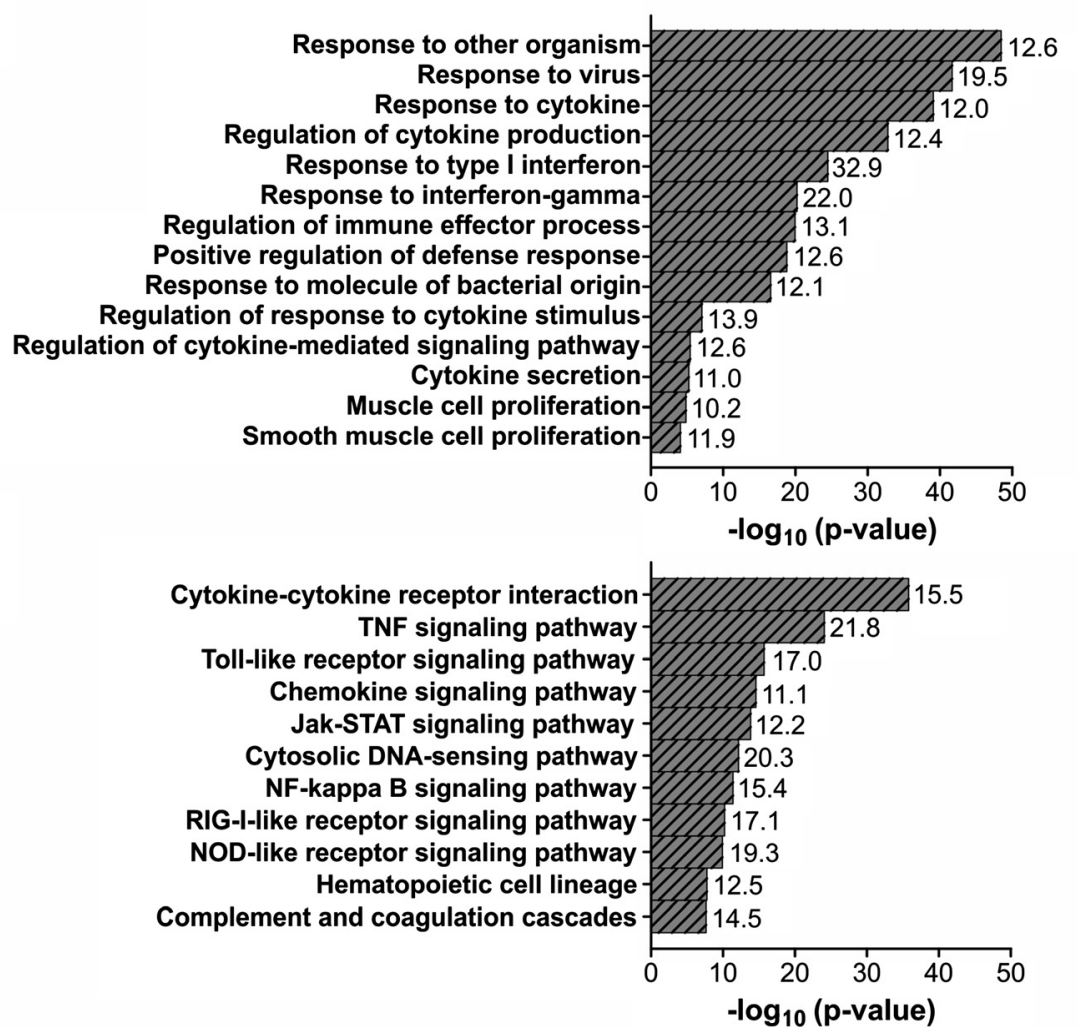

Fig. 2 Common changes in cellular gene expression after RV infection in fetal and adult endothelial cells. a Displayed are the percent of genes that exhibited expression changes greater than or equal to 4-fold with an ANOVA p-value of $\leq 0.01$. The percentage is based on the total amount of genes assayed. $\mathbf{b}$ Venn diagram showing the intersection between the up-regulated $(\uparrow)$, down-regulated $(\downarrow)$ and contra-egulated $(\uparrow)$ transcripts $36 \mathrm{~h}$ after RV infection (MOI of 10) of HUVEC and HSaVEC. c Biological process and d KEGG pathway analysis of the 392 transcripts that were affected after RV infection in both fetal and adult endothelial cells. Displayed are the ranked Bonferroni corrected p-values of significantly enriched biological processes and pathways $(p \leq 0.01)$. Related terms were grouped and the most significant term of the group was defined as the group leading term. Numbers at the bar represent genes (in \%) from the cluster that were associated with the term 
relative modulation of cellular transcription induced by RV infection. Of the 38,500 human genes assayed, 834 $(2.2 \%)$ and $769(2.0 \%)$ genes were differentially regulated in HUVEC and HSaVEC, respectively (Fig. 2 (a)). In HUVEC, 563 genes were up-regulated and 271 were down-regulated following RV infection. Similarly, in HSaVEC, 621 genes were up-regulated and 148 were down-regulated. A comparison of the number of differentially regulated genes in both endothelial cells showed an overlap of more than 75 \% (Fig. 2 (b)). Of the overlapping genes, five genes were contra-regulated. Remarkably, a larger proportion of genes were down-regulated in HUVEC after RV infection compared to HSaVEC.
Due to the high overlap of genes which are affected by RV infection in HUVEC and HSaVEC, we wanted to know whether certain biological processes and cellular pathways were statistically overrepresented. GO term-based analysis of the 392 commonly regulated genes was applied. As shown in Fig. 2 (c), fourteen biological processes were enriched in the endothelial cells. Remarkably, a large proportion of genes were assigned to $\mathrm{GO}$ terms involved in cytokine production and regulation. Furthermore, GO terms involved in cellular defense mechanisms were enriched in infected HUVEC and HSaVEC. In addition to GO termanalysis, KEGG pathway analysis (Fig. 2 (d)) revealed

Table 1 Differentially expressed genes of the chemokine family

\begin{tabular}{|c|c|c|c|}
\hline \multirow[t]{2}{*}{ Gene symbol } & \multirow[t]{2}{*}{ Gene title } & \multicolumn{2}{|c|}{ Mean fold change ${ }^{(a)}$} \\
\hline & & HUVEC & HSaVEC \\
\hline CCL2 & chemokine (C-C motif) ligand 2 & 21.03 & n.s. \\
\hline CCL2O & chemokine (C-C motif) ligand 20 & 112.23 & 122.14 \\
\hline CCL23 & chemokine (C-C motif) ligand 23 & 4.32 & 4.09 \\
\hline CCL3, CCL3L1, CCL3L3 & $\begin{array}{l}\text { chemokine (C-C motif) ligand 3; } \\
\text { chemokine (C-C motif) ligand 3-like } \\
\text { 1; chemokine (C-C motif) ligand 3-like } 3\end{array}$ & 194.09 & 278.09 \\
\hline CCL4 & chemokine (C-C motif) ligand 4 & 231.15 & 972.27 \\
\hline \multirow[t]{3}{*}{ CCL5 } & chemokine ( $\mathrm{C}-\mathrm{C}$ motif) ligand 5 & 1394.87 & 430.3 \\
\hline & & 1254.72 & 325.72 \\
\hline & & 414.38 & 310.81 \\
\hline CCL7 & chemokine (C-C motif) ligand 7 & 115.92 & n.s. \\
\hline CCL8 & chemokine ( $\mathrm{C}-\mathrm{C}$ motif) ligand 8 & 579.29 & 151.42 \\
\hline \multirow[t]{2}{*}{ CX3CL1 } & chemokine ( $\mathrm{C}-\mathrm{X} 3-\mathrm{C}$ motif) ligand 1 & 23.37 & 36.99 \\
\hline & & 12.43 & 28.53 \\
\hline CXCL1 & chemokine (C-X-C motif) ligand 1 & 5.59 & n.s. \\
\hline CXCL10 & chemokine (C-X-C motif) ligand 10 & 4833.33 & 297.78 \\
\hline CXCL11 & chemokine (C-X-C motif) ligand 11 & 382.38 & 72.25 \\
\hline \multirow[t]{2}{*}{ CXCL12 } & chemokine (C-X-C motif) ligand 12 & 35.66 & n.s. \\
\hline & & 7.43 & \\
\hline CXCL16 & chemokine (C-X-C motif) ligand 16 & 8.68 & 6.29 \\
\hline \multirow[t]{3}{*}{ CXCL2 } & chemokine (C-X-C motif) ligand 2 & 28.56 & 4.70 \\
\hline & & 25.62 & 4.18 \\
\hline & & 22.74 & \\
\hline CXCL3 & chemokine (C-X-C motif) ligand 3 & 92.97 & 33.14 \\
\hline \multirow[t]{3}{*}{ CXCL5 } & chemokine (C-X-C motif) ligand 5 & 44.83 & 15.34 \\
\hline & & 22.63 & 12.55 \\
\hline & & & 12.1 \\
\hline CXCL6 & chemokine (C-X-C motif) ligand 6 & 84.27 & 8.97 \\
\hline CXCL9 & chemokine (C-X-C motif) ligand 9 & 41.56 & 88.71 \\
\hline
\end{tabular}

${ }^{(a)}$ Displayed are average fold change of RV-infected cells in comparison to non-infected cells detected by transcriptome analysis. In positions that are labeled n.s., the gene expression did not meet the selected criteria (i.e. fold change cut-off $\leq-4$ and $\geq 4$ and ANOVA p-value of $\leq 0.01$ ). If a gene was detected by several different probes, all fold changes that meet the selected criteria are shown 
the enrichment of pathways which are involved in antiviral responses, such as the Toll-like receptor signaling pathway and the RIG-I-like receptor signaling pathway.

Similar chemokine expression pattern after RV infection in fetal and adult endothelial cells

Chemokines are chemotactic cytokines that play an important role during inflammation and homeostasis. While inflammatory chemokines are required to recruit immune cells to the site of infection, homeostatic chemokines possess a key function in controlling cell migration during developmental processes. Interference with the precisely coordinated production of chemokines triggered by a viral infection has profound effects on fetal development and is speculated to contribute to the pathological findings of CRS [14].

Our GO term analysis revealed that biological processes involved in cytokine production, regulation, and processing are enriched in both adult and fetal primary endothelial cells. Moreover, gene expression was strongly increased for the interferon-inducible chemokines (Table 1). To verify up-regulation of these chemokines on the protein level, HUVEC and HSaVEC were infected at an MOI of 10 with RV and the chemokine levels in the supernatant were quantified with a protein array at 48 hpi (Fig. 3). Similar to the microarray results, significantly increased amounts of CCL2, CCL3, CCL4, CCL5,

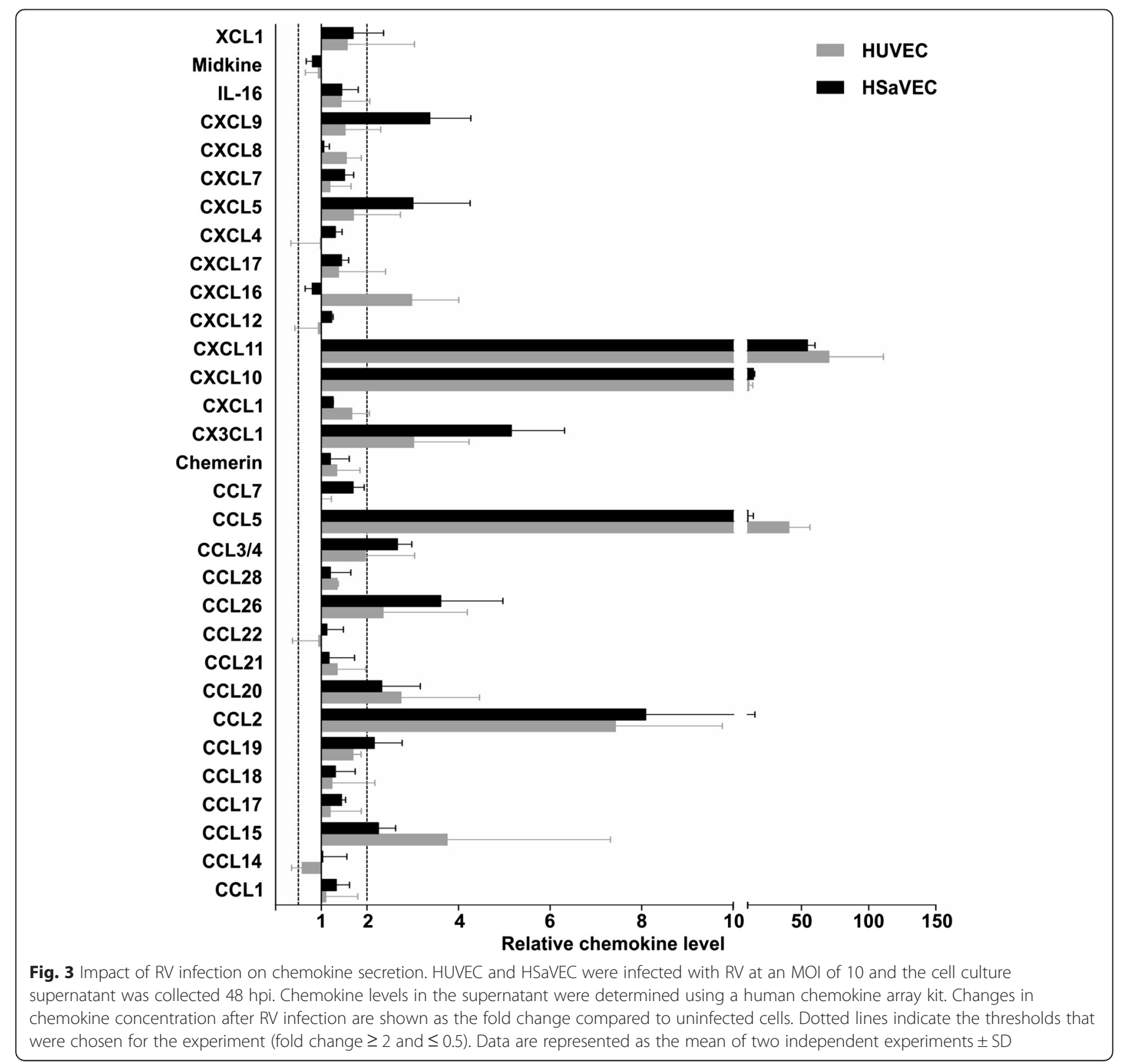


CCL15, CCL20, CCL26, CXCL10, CXCL11 and CX3CL1 were detected in the supernatant following RV infection in both HUVEC and HSaVEC. The overall pattern of chemokine production before and after infection was similar in the endothelial cells (Additional file 2). Interestingly, one chemokine, CCL14, appeared to be secreted at significantly lower amounts into the supernatant following RV infection of HUVEC.

Identification of differentially regulated genes induced by RV in fetal and adult cells

In order to identify biological processes which are differentially regulated after RV infection of primary cells of adult and fetal origin, GO term-based cluster analysis was carried out with genes that were up- or downregulated following infection compared to non-infected cells. Figure 4 shows the GO terms that were significantly $(\mathrm{p} \leq 0.01)$ over-represented in the set of genes that were up- or down-regulated after RV infection of HUVEC and HSaVEC. Displayed are the Bonferroni corrected p-values, with the numbers at the bar representing the percentage of genes from the cluster that were associated with the term (compared with all genes associated with the term). In addition to genes involved in immune defense processes, genes associated with angiogenesis and vascular development were over-represented following RV infection in the up-regulated gene list of HSaVEC. No GO terms were over-represented in the down-regulated gene list of HSaVEC. In the set of genes up-regulated in HUVEC following RV infection, GO terms involved in immune processes were overrepresented. Moreover, the GO term "negative regulation of immune effector processes" was also over-represented in this set of genes, indicating that genes are activated upon infection that counteract the cellular immune response. Interestingly, 18 genes assigned to the GO term "sensory organ development", "eye development", and "ear development" were over-represented in the set of down-regulated genes of HUVEC, but not HSaVEC. Heart malformation, deafness and ocular conditions such as cataracts are the most common clinical manifestations of CRS, however the molecular mechanisms that lead to the pathological conditions (or the gene products involved) are not known. Table 2 summarizes the fold

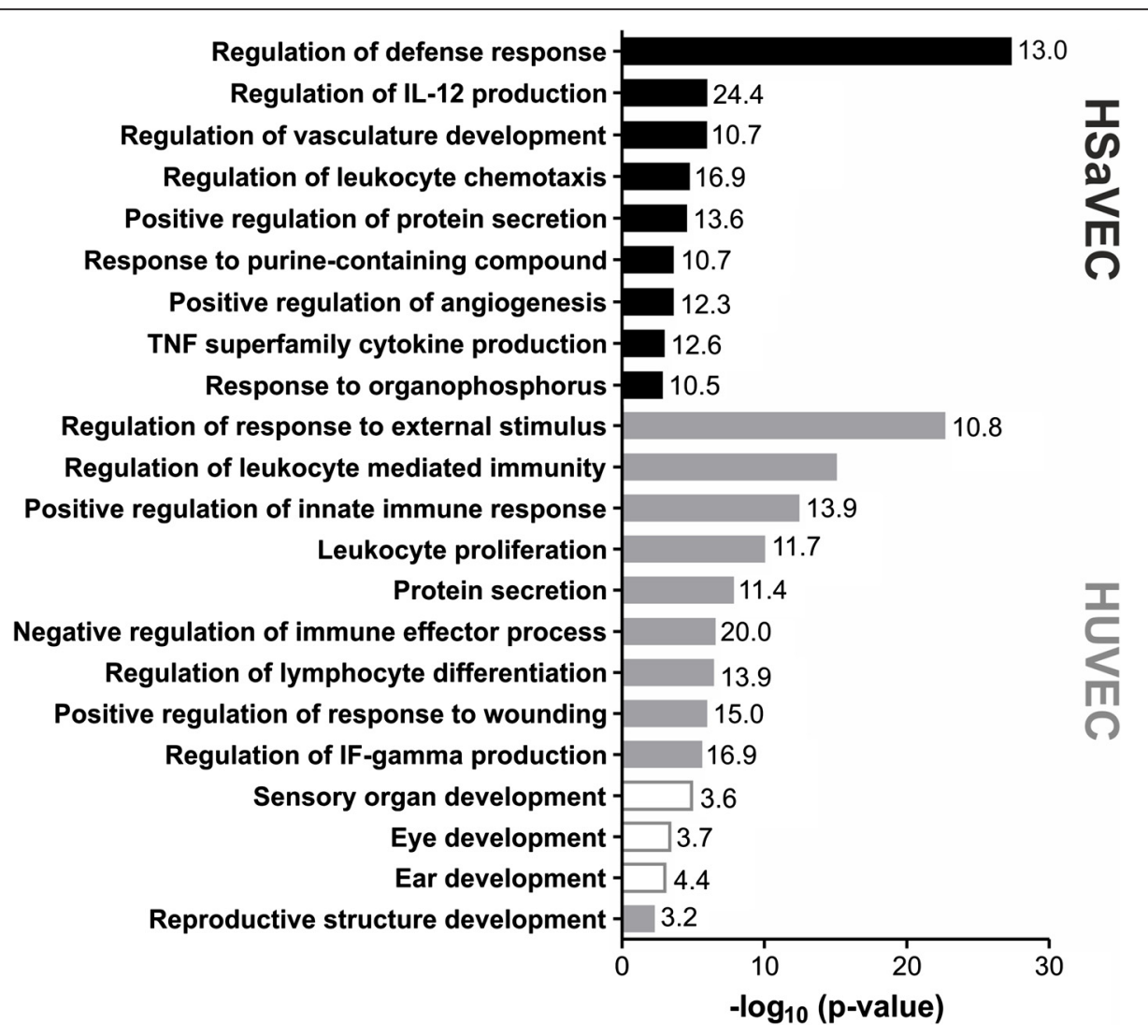

Fig. 4 Bonferroni corrected p-values of significantly enriched biological processes which are unique to the two primary cells following RV infection. Related terms were merged into functional groups and the most significant term was defined as the group-leading term. Numbers at the bar represent genes (in \%) from the cluster that were associated with the term. Unfilled bars indicate if the GO term is derived from the downregulated gene list of HUVEC. Filled bars indicate if the GO term is derived from the up-regulated gene list of HUVEC (grey) and HSaVEC (black) 
Table 2 Differential expression of genes belonging to the GO term "sensory organ development" in HUVEC and HSaVEC following RV infection

\begin{tabular}{|c|c|c|c|}
\hline \multirow[t]{2}{*}{ Gene symbol } & \multirow[t]{2}{*}{ Gene title } & \multicolumn{2}{|c|}{ Fold change microarray ${ }^{(a)}$} \\
\hline & & HUVEC & HSaVEC \\
\hline ADAMTS18 & $\begin{array}{l}\text { ADAM metallopeptidase with thrombospondin } \\
\text { type } 1 \text { motif, } 18\end{array}$ & -4.89 & n.s. \\
\hline ALDH1A2 & aldehyde dehydrogenase 1 family, member A2 & -4.16 & n.s. \\
\hline CLN8 & ceroid-lipofuscinosis, neuronal 8 & -8.05 & n.s. \\
\hline DSCAM & Down syndrome cell adhesion molecule & -9.98 & n.s. \\
\hline FGFR2 & fibroblast growth factor receptor 2 & -10.01 & 5.03 \\
\hline FZD3 & frizzled family receptor 3 & -10.00 & -8.56 \\
\hline \multirow[t]{2}{*}{$J A G 2$} & \multirow[t]{2}{*}{ jagged 2} & -4.86 & \multirow[t]{2}{*}{ n.s. } \\
\hline & & -4.01 & \\
\hline MYOЗА & myosin $3 \mathrm{~A}$ & -9.46 & n.s. \\
\hline MYOTA & myosin 7A & -5.65 & -6.17 \\
\hline NHS & Nance-Horan syndrome & -4.35 & n.s. \\
\hline NOG & noggin & -6.68 & -5.04 \\
\hline NTRK3 & neurotrophic tyrosine kinase, receptor, type 3 & -7.70 & n.s. \\
\hline PDGFRA & $\begin{array}{l}\text { platelet-derived growth factor receptor, alpha } \\
\text { polypeptide }\end{array}$ & -6.24 & n.s. \\
\hline RDH10 & retinol dehydrogenase 10 (all-trans) & -9.00 & n.s. \\
\hline SLC25A27 & solute carrier family 25, member 27 & -16.78 & n.s. \\
\hline TNPO1 & transportin 1 & -7.66 & n.s. \\
\hline TRPM1 & $\begin{array}{l}\text { transient receptor potential cation channel, } \\
\text { subfamily } \mathrm{M} \text {, member } 1\end{array}$ & -7.72 & -5.76 \\
\hline TSPAN12 & tetraspanin 12 & -4.42 & n.s. \\
\hline
\end{tabular}

${ }^{a}$ Displayed are the average fold changes from three independent experiments of RV-infected cells in comparison to non-infected cells detected by the microarray that meet the selected criteria (i.e. fold change cut-off $\leq-4$ and $\geq 4$ and ANOVA p-value of $\leq 0.01$ ). If a gene was detected by several different probes, all fold changes that meet the selected criteria are shown. Positions labeled n.s. where not shown to be significantly up- or down-regulated in the DNA microarray analysis

changes of the down-regulated sensory organ development genes as detected by microarray analysis for HUVEC and HSaVEC. Four of the 18 genes that were downregulated in HUVEC were also down-regulated in $\mathrm{HSa}$ VEC. The remaining 14 genes were either unaffected or up-regulated following infection in these cells.

In order to elucidate the putative molecular processes leading to pathogenesis, we sought to verify the downregulated expression of genes assigned to the GO term "sensory organ development" by qPCR. Therefore, HUVEC and HSaVEC were infected with Wb-12 at an MOI of ten and total RNA was isolated $36 \mathrm{hpi}$ and subjected to qPCR. For HUVEC, two pools of cells were utilized for five independent experiments; whereas for HSaVEC, two donors were used for six independent experiments. Non-infected cells served as the control and $G A P D H$ was used for normalization. Analysis of gene expression by qPCR was carried out with 13 genes according to the $\triangle \Delta \mathrm{CT}$ method. Of the 13 genes belonging to this GO term, qPCR was able to confirm the downregulation of CLN8, FGFR2, FZD3, JAG2, MYO7A, NHS,
NOG and SLC25A27 in infected HUVEC (Fig. 5). Downregulation was verified for FZD3, NOG and SLC25A27 in infected HSaVEC; the other ten sensory organ development genes were either up-regulated or not affected in this cell type.

Differences in gene expression between HUVEC and HSaVEC following RV infection might arise due to significant differences in basal gene expression. In order to rule this out for the selected sensory organ development genes, the basal expression levels of non-infected endothelial cells were compared with each other (Table 3). Based on our selected criteria (i.e. fold change cut-off $\leq-4$ and $\geq 4$ and ANOVA p-value of $\leq 0.01$ ) only the basal gene expression level of ADAMTS18 was significantly lower and NHS was significantly higher in HUVEC compared to HSaVEC. Thus, we cannot completely rule out for these genes that the observed differences following RV infection between the primary endothelial cells might be the result of differences in basal expression rates. However, for the remaining eleven other genes examined, no differences in basal gene expression were seen. 


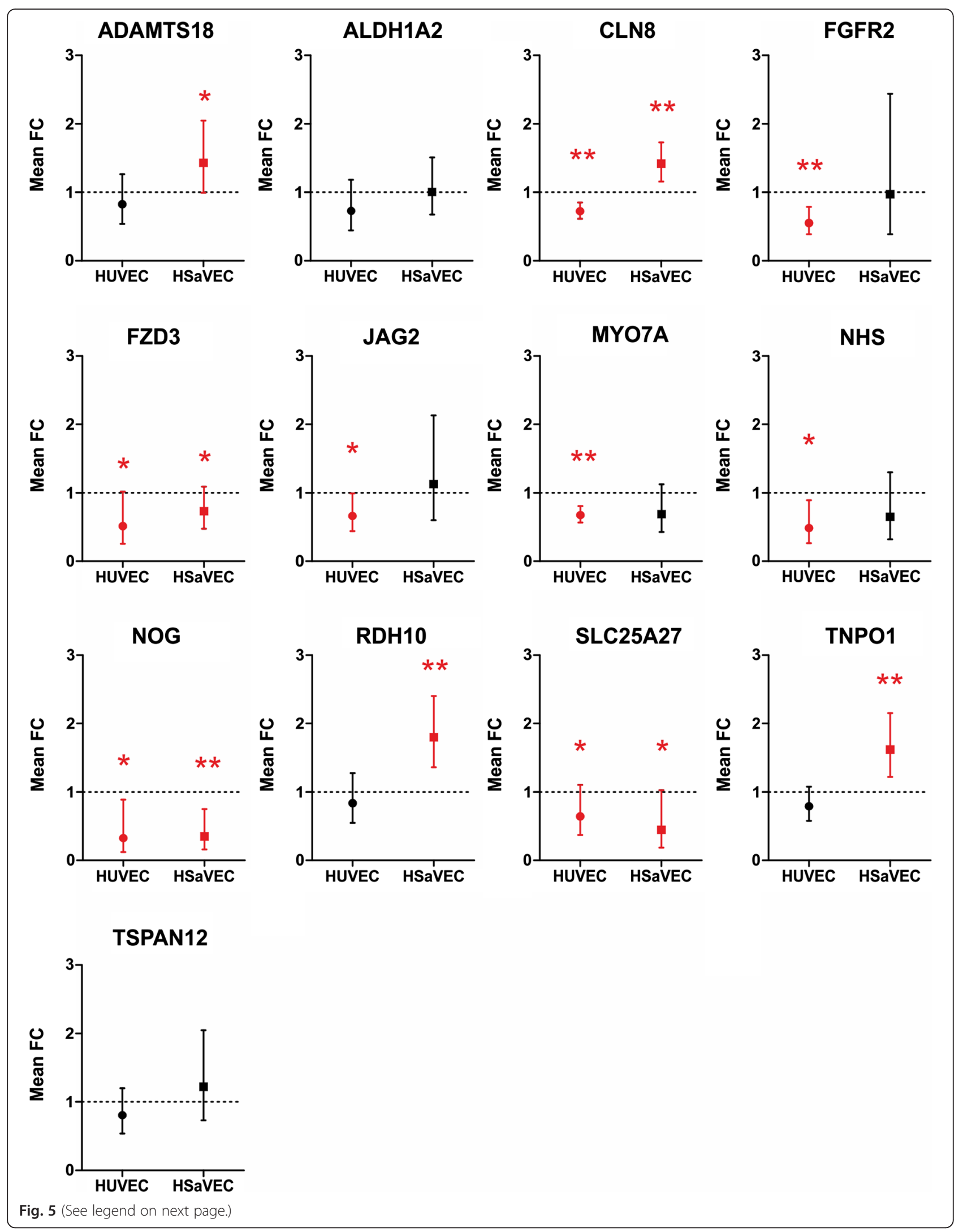


(See figure on previous page.)

Fig. 5 Differential expression of genes belonging to the GO term "sensory organ development" in HUVEC and HSaVEC following RV infection as determined by qPCR. HUVEC and HSaVEC from different donors were infected with RV at an MOI of 5 and gene expression relative to noninfected cells was quantified by qPCR 36 hpi. GAPDH was used for normalization. Bars denote mean fold change (FC, $n=5$ for HUVEC and $n=6$ for HSaVEC) with confidence intervals calculated by the $\triangle \triangle C T$ mathematical model. A one-tailed paired t-test was used to determine statistically significant differences in gene expression compared to non-infected cells using $\Delta C T$ values and is indicated by an asterisk and red coloring $\left({ }^{*}, p \leq 0.05 ;{ }^{* *}, p \leq 0.01\right)$

Primary cells are thought to be a good model for studying viral infections. However, these cells have been shown to exhibit significant donor-to-donor variability in some biological aspects. Although two pools of HUVEC and HSaVEC from two different donors were used for $\mathrm{qPCR}$, we wanted to ensure that the observed effects are not unique to a certain donor. Thus, we compared the levels of sensory organ gene expression following RV infection with cells from different donors. Fig. 6 compares the $\triangle \Delta C T$ values obtained from infections with two pools of HUVEC - with each pool of cells consisting of three different donors. The expression of FZD3, NHS, NOG and TSPAN12 differed significantly between the two pools of cells following infection. Despite the significant difference, the direction of regulation (i.e. down-regulation) was the same for FZD3, NHS and NOG between the pools of cells. Thus, down-regulation of the sensory organ development genes could be observed for the majority of genes in HUVEC derived from different donors, suggesting that the observed effects on expression were not donor specific.

Since pooled HSaVEC are not commercially available and the number of donors is limited, the expression changes for two individual donors were compared and showed a significant difference between donor 1 and 2 for NHS. However, the remaining twelve sensory organ development genes did not differ between the two donors following RV infection (Fig. 7).

\section{Discussion}

Cardiovascular abnormalities, sensorineural hearing loss, cataracts, and glaucoma have been the most prominent findings among patients suffering from CRS [8]. Although $\mathrm{RV}$ has been shown to efficiently replicate in endothelial cells [19], and was also found in the endothelium of infected fetuses $[6,7,9]$, studies with human endothelial cells are scarce. Most research was conducted with nonhuman cell lines, such as Vero, BHK and RK13.

Since it has been speculated that endothelial cells play an important role in the birth defects associated with CRS, we wanted to explore the effect of RV infection on primary human endothelial cells of adult and fetal origin by gene expression analysis. HUVEC are considered fetal cells because they are derived from the umbilical cord veins, which develop genetically and physiologically from the fetus. In contrast to that, HSaVEC are derived from the saphenous vein of adult donors. By analyzing the effects of RV replication on fetal and adult endothelial cells we approched to understand the mechanisms underlying $\mathrm{RV}$-induced teratogenesis.

Efficient replication of RV in endothelial cells was previously reported for HUVEC [19], but not for HSaVEC. Therefore, the primary endothelial cells were analyzed for their ability to support viral replication, in addition to the Vero76 and A549 cell lines (which have been routinely used to cultivate RV). The replication kinetics and the detection of viral proteins showed that RV replicates both in fetal and adult primary endothelial cells. RV infection of HUVEC resulted in slightly lower extracellular titers compared to the infection of the immortalized cell lines Vero76 and A549. The extracellular titers after infection of HSaVEC were about 10 -fold reduced, which might be caused by the slower growth rate of this cell line. However, viral titers remained almost constant for the observation period of five days after infection with an MOI of five, indicating that the virus caused a persistent infection in the immortalized cell lines as well as in the primary endothelial cells. The results of the capsid expression analysis show that the immortalized cell lines Vero76 and A549 are infected more efficiently by RV than HUVEC and HSaVEC by a factor of almost two. Synchronous infection has not been observed in any of the cell lines after infection at an MOI of

Table 3 Basal expression of genes belonging to the GO term "sensory organ development" in HUVEC and HSaVEC

\begin{tabular}{llcc}
$\begin{array}{l}\text { Gene } \\
\text { symbol }\end{array}$ & Gene title & $\begin{array}{l}\text { Fold Change HUVEC vs } \\
\text { HSaVEC }^{(a)}\end{array}$ & $\begin{array}{l}\text { ANOVA p-value HUVEC vs } \\
\text { HSaVEC }^{(b)}\end{array}$ \\
\hline ADAMTS18 & ADAM metallopeptidase with thrombospondin type 1 motif, & -5.87 & 0.000027 \\
& 18 & 5.43 & 0.000766 \\
\hline
\end{tabular}

(a) Displayed are the average fold changes from three independent experiments of non-infected HUVEC in comparison to non-infected HSaVEC detected by microarray that meet the selected criteria (i.e. fold change cut-off $\leq-4$ and $\geq 4$ and ANOVA $p$-value of $\leq 0.01$ )

(b) ANOVA p-values $\leq 0.01$ are considered significant 


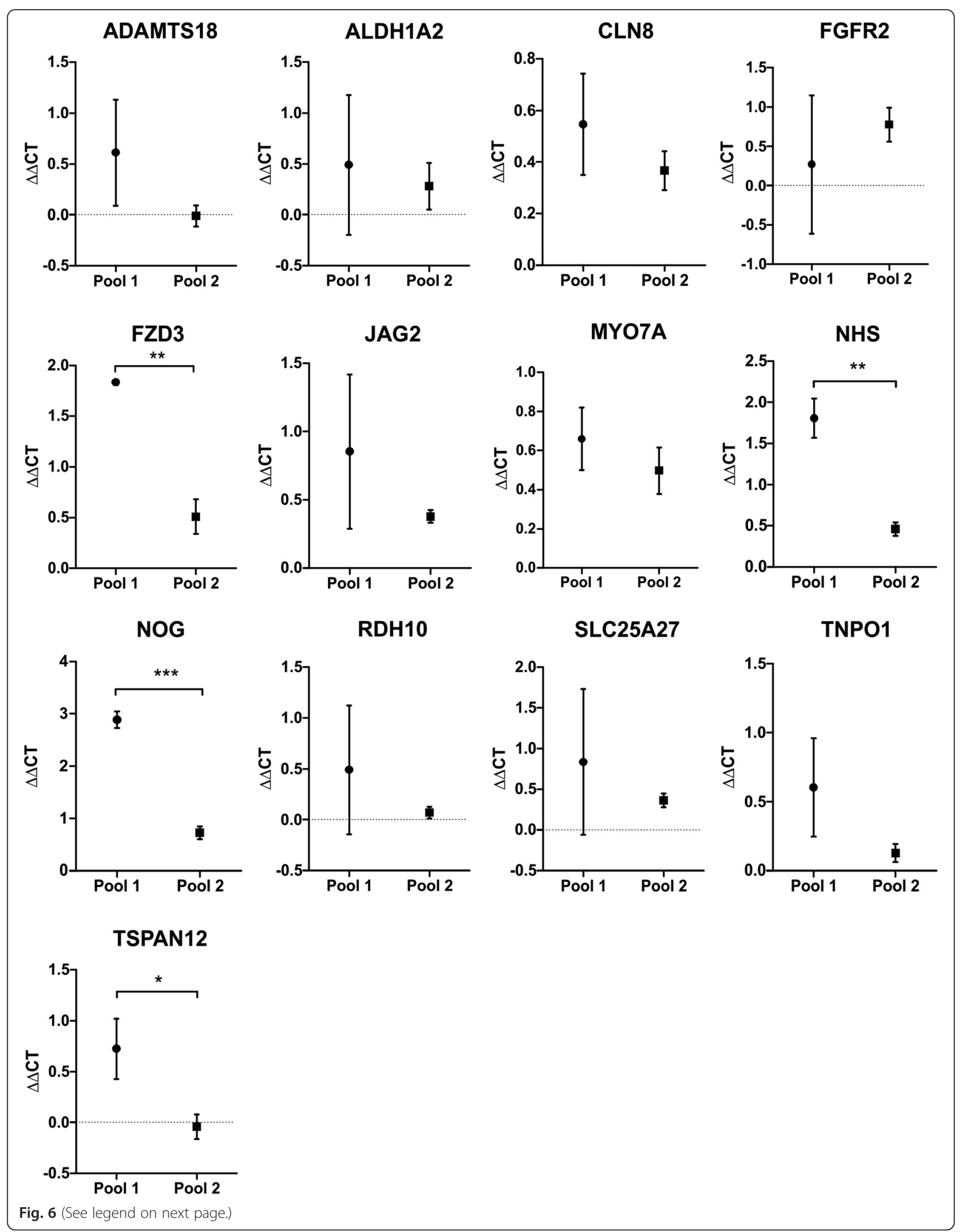


(See figure on previous page.)

Fig. 6 Donor-to-donor variation of sensory organ gene expression in HUVEC isolated from different donors. Two pools of HUVEC, with each pool consisting of cells from three different donors, were infected with RV at an $\mathrm{MOI}$ of 5 and gene expression relative to non-infected cells was quantified by qPCR 36 hpi. GAPDH was used for normalization. Bars denote mean $\triangle \triangle C T \pm$ SEM with $\triangle \Delta C T$ values $<0$ indicating up-regulation and $\Delta \Delta C T$ values $>0$ indicating down-regulation. A two-tailed unpaired t-test was used to determine statistically significant differences in gene expression using $\Delta C T$ values and is indicated by an asterisk (*, $p \leq 0.05{ }^{* *}, p \leq 0.01$ )

5 , which is consistent with findings in other various cell lines $[14,17,18,21]$. In one other study, infection of HUVEC with a clinical isolate of a different genotype (1E) has been shown to be synchronous at a high MOI [19]. Possibly, different RV strains may have distinct efficiencies and properties regarding infection or different cellular tropisms. Since RV-infected HUVEC and HSaVEC with similar efficiencies, it is reasonable to assume that the virus generally does not have a stronger tropism for fetal cells than for adult cells.

The aim of the present study was to identify differences in the gene expression pattern following RV infection in fetal and adult primary cells in order to understand the teratogenicity of the virus. More than $75 \%$ of the differentially regulated genes following RV infection overlapped between HUVEC and HSaVEC cells indicating similar gene expression modulation following RV infection. Not surprisingly, these genes were grouped to GO terms involved in host defense mechanisms, such as cytokine regulation and production. M. P. Adamo, et al. [14] and X. Y. Mo, et al. [15] reported a robust interferon-stimulated gene response following infection of human embryonic fibroblasts and the ECV304 cell line, respectively. This is corroborated by our findings, since expression of interferon-inducible genes, such as $O A S L$ and $M X 2$, was strongly upregulated following RV infection. Moreover, these genes showed the highest ranking position when the commonly expressed genes of HUVEC and HSaVEC were analyzed (Additional file 3).

It has been speculated that dysregulated expression of cytokines and chemokines following RV infection disrupts cellular processes in the developing fetus, thereby contributing to CRS. However, data on which chemokines are produced during RV infection and how they affect the developing fetus are missing. Using the primary endothelial cell model system, we examined whether increased expression of chemotactic cytokines was detected on the protein level by a chemokine array. In concordance with the microarray results, an increase of inflammatory chemokines was detected in the supernatant of the RV-infected endothelial cells. Interestingly, CCL5, CXCL10, and CXCL11 - which have been reported to play a key role in the pathogenesis of cardiovascular disease [22-24] - appeared to be most upregulated (more than 10-fold). CCL14 was the only chemokine tested in the protein array for which a reduction was seen after infection in HUVEC, but not in
HSaVEC. CCL14 was also down-regulated 2.31 fold (ANOVA p-value 5.27E-14) in the transcriptional profiling analysis after infection of HUVEC. Interestingly, CCL14 is highly expressed during early pregnancy by the human endometrium [25] and has been implicated in regulating the implantation of the embryo at the fetalmaternal interface. The corresponding receptor of CCL14, CCR1, which is mainly expressed by trophoblasts, was also down-regulated 5.70-fold (ANOVA p-value 6.96E-03) as detected by microarray analysis in HUVEC after RV infection. Thus, perturbation of this chemokine-receptor network might interfere with maternal-fetal communication and contribute to RV-associated embryo resorption or disturbed placental and fetal development. Interestingly, this receptor was also found to be down-regulated following infection of monocytes with another teratogen, HCMV [26]. However, the role of RV infection in vivo on CCL14 and CCR1 expression requires further investigation.

The most prominent clinical manifestations observed in CRS patients are impairment in vision and hearing as well as cardiovascular abnormalities. As of today, it is not understood which mechanisms contribute to this symptomatology or which genes are involved in the RVinduced pathophysiology. However, dysfunction of the sensory organs has been speculated to be a result of vascular insufficiency causing nutrient deprivation rather than being caused by direct viral damage [3]. By examining differences in gene expression between endothelial cells of fetal and adult origin, we wanted to investigate which genes might contribute to the RV-induced pathophysiology in the fetus. GO term-based cluster analysis of the down-regulated genes revealed that terms belonging to sensory organ development (GO term "sensory organ development", "ear development", "eye development") were only enriched for HUVEC. Gene expression analysis by qPCR of RV-infected HUVEC also showed a significant down-regulation of eight genes belonging to this GO term.

While all genes encode proteins that possess functions in sensory organ development, their molecular functions and associated diseases are diverse. The genes ceroidlipofuscinosis, neuronal $8(C L N 8)$ and $N H S$ play a role during sensory organ development of the eye: CLN8 has a function in lipid synthesis and transport and mutations are associated with progressive epilepsy, mental retardation, and vison impairment [27]. Mutations in the NHS gene are associated with Nance-Horan-Syndrome, characterized 


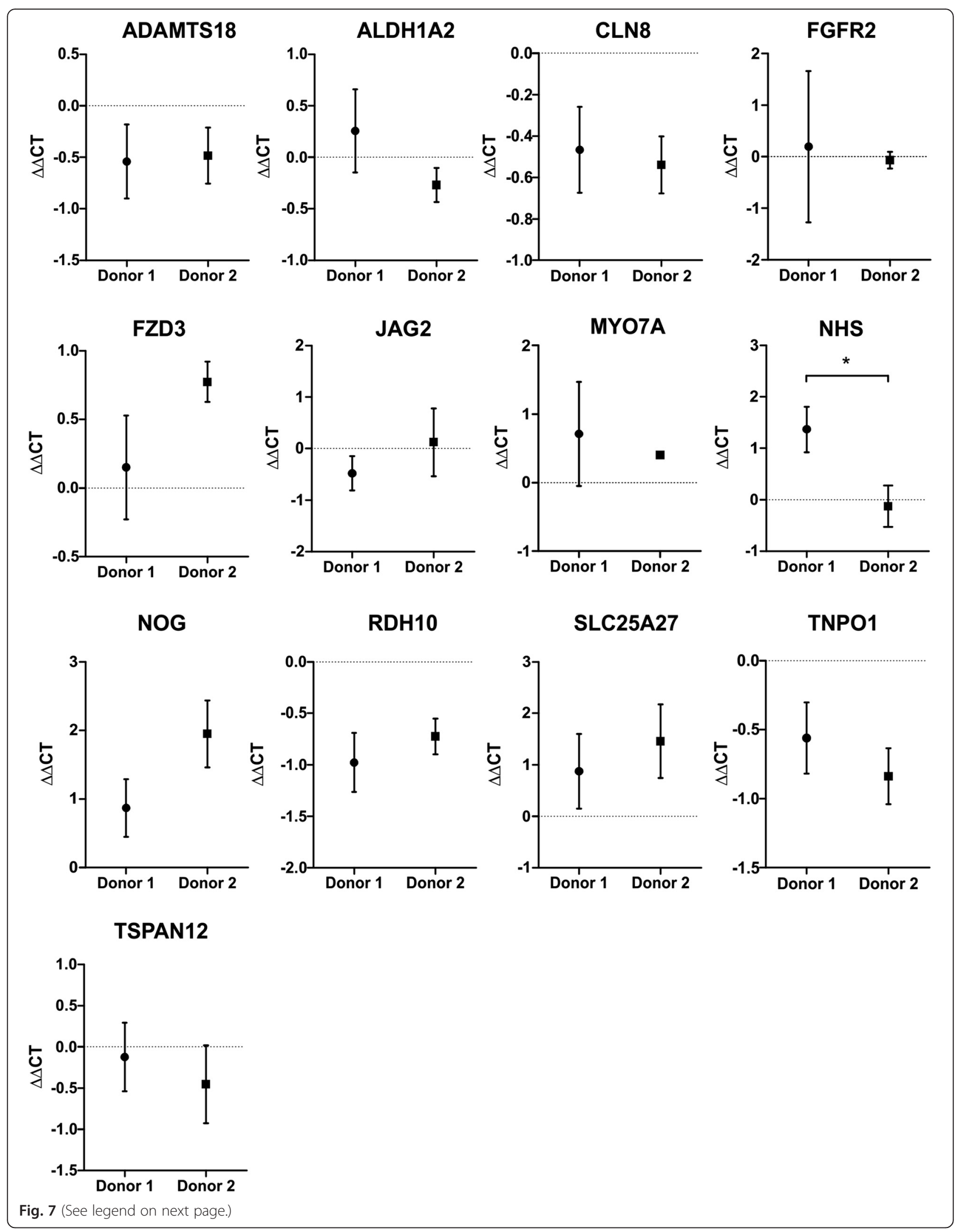


(See figure on previous page.)

Fig. 7 Donor-to-donor variation of sensory organ gene expression in HSaVEC isolated from two different donors. HSaVEC obtained from two different donors were infected with RV at an MOI of 5 and gene expression relative to non-infected cells was quantified by qPCR 36 hpi. GAPDH was used for normalization. Bars denote mean $\triangle \triangle C T \pm$ SEM with $\triangle \triangle C T$ values $<0$ indicating up-regulation and $\Delta \triangle C T$ values $>0$ indicating downregulation. A two-tailed unpaired $t$-test was used to determine statistically significant differences in gene expression using $\Delta C T$ values and is indicated by an asterisk (*, $p \leq 0.05)$

by ocular abnormalities such as cataracts. It was demonstrated that the NHS protein is a regulator of actin remodeling, and it was speculated that its function is required for maintaining cell morphology during embryogenesis [28].

The genes FZD3, JAG2, NOG and SLC25A27, encode for proteins that are attributed to ear development. The function of FZD3 protein is still under debate, but recent data indicate that FZD3 is involved in regulating axonal navigation during embryonic development [29]. The putative Notch-ligand, JAG2, was shown to control the number of sensory hair cells that form the organ of corti in the cochlea of the inner ear $[30,31]$. The gene product of $N O G$ is required for neural tube growth and patterning, but was also demonstrated to interfere with bone morphogenetic protein (BMP) signaling, thereby blocking sensory organ morphogenesis of the inner ear in different animal models [32-34]. The function of the mitochondria uncoupling protein (UCP4), encoded by SLC25A27, is still a matter of debate; however, expression of this protein has been found in neurosensory cells of the inner ear supporting the hypothesis that UCP4 plays a role in functional maturation of the inner ear [35].

The gene product of FGFR2 and MYO7A are suspected to function both in ear and eye development. Mutations in the FGFR2 gene have been associated with skeletal abnormalities [36] and Lacrimo-auriculo-dento-digital (LADD [MIM 149730]) syndrome, a disorder characterized by hearing loss, deformity in the network of structures of the eye, lacrimal-duct aplasia, and malformations of teeth, forearms, and fingers. Defects in MYO7A have been shown to cause Usher syndrome, a disorder characterized by sensorineural hearing loss combined with retinitis pigmentosa $[37,38]$.

FZD3, NOG and SLC25A27 were also shown to be down-regulated in HSaVEC, indicating that the regulation of these genes seems to be independent of cell origin. Nevertheless, one could imagine that interference with the expression of these three genes during embryonic development would have a profound effect on the organism, whereas the effect would not be as dramatic in the adult. In contrast, ADAMTS18, CLN8, RDH10 and TNPO1 were shown to be up-regulated following infection in HSaVEC, indicating that gene regulation differs for these genes between the endothelial cells.

Development of organs belonging to the sensory system is complex and must be precisely coordinated and controlled by intrinsic and extrinsic factors. Interference with the expression of genes involved in these processes may be fatal during embryogenesis and, furthermore, the down-regulation of these genes may give some explanations as to why RV infection is often associated with vision and hearing loss, but causes only mild and transient symptoms in patients infected postnatally. Interestingly, in a study by M. P. Adamo, et al. [14], the here detected down-regulated genes belonging to the GO term "sensory organ development" were not shown to be differentially regulated in their transcriptome analysis of the RV-infected human embryonic fibroblasts (HEF).

In order to the see common and different patterns in gene expression between our primary cells and the cell lines used in the transcriptome analysis carried out by $\mathrm{M}$. P. Adamo, et al. [14], the gene lists of the cells of fetal and adult origin from both studies were compared with each other (Additional file 4 (a)). In the study of M. P. Adamo, et al. [14] gene expression of primary human embryo fibroblasts (HEF) was compared with a fibroblast cell line from the human adult lung (Hs888Lu) following RV infection. Interestingly, when comparing the up-and downregulated genes between the HUVEC, HSaVEC, Hs888Lu, and HEF, only a small overlap was detected. 86 genes were commonly up-regulated in HUVEC and HEF, whereas only 56 genes were commonly up-regulated in HSaVEC and Hs888Lu. When comparing the down-regulated genes, the overlap was even smaller with eight and three commonly down-regulated genes for fetal and adult cells, respectively. Not surprisingly, the commonly upregulated genes of fetal cells showed an enrichment of GO-terms involved in viral defense mechanisms (Additional file 4 (b)). For the other commonly regulated genes, no GO-terms were enriched. However, most of the up-regulated genes of adult cells also contained pro-inflammatory genes involved in viral defense mechanisms. Many antiviral strategies are switched on upon viral infection and are conserved, even if different viruses and cell types are analyzed. Thus, it is not surprising that antiviral genes were up-regulated in the endothelial cells as well as HEF and Hs888Lu. However, a down-regulation of genes belonging to the GO term "sensory organ development" was only observed in our study in HUVEC. Our use of a different virus strain and a later harvest time, but more importantly the use of a different cell type, might be responsible for this observation. Since damage to the vascular endothelium has been observed in RVinfected fetuses, we believe that our primary endothelial 
cell model is well suited for studying RV teratogenesis and we speculate that the prominent clinical manifestations observed in CRS patients arise due to the deregulation of sensory organ development genes in endothelial cells.

\section{Conclusion}

The data presented here show that RV infection perturbs the gene expression of its host. While most RV-induced changes in transcription are common to endothelial cells of fetal and adult origin (including those involved in antiviral processes), we also detected unique gene expression changes depending on the cell type. Most remarkably, a set of genes involved in sensory organ development was down-regulated in the primary cells of fetal origin. Since we could show that RV efficiently replicates in the primary endothelial cells and since lesions in the endothelium are a prominent finding in histopathological studies of CRS fetuses, we propose that replication of RV in the fetal endothelium leads to a downregulation of genes required for ear and eye development. It still remains unknown, however, which viral gene products and cellular transcription factors are responsible for the down-regulation of these genes. Nevertheless, the down-regulation was mainly observed in the primary endothelial cells of fetal origin. Thus, we believe that the special cellular environment during fetal and embryonic development plays a major role in this process and further studies should be conducted with primary fetal endothelial cells, such as HUVEC, rather than non-fetal cell lines.

\section{Methods}

\section{Cell culture and viruses}

Two pools of HUVEC (from three donors, Promocell) and HSaVEC from two single donors, Promocell), between passage three and eight, were cultured in Endothelial Cell Growth Medium 2 (Promocell), supplemented with $100 \mu \mathrm{g} / \mathrm{ml}$ streptomycin and $100 \mathrm{U} / \mathrm{ml}$ penicillin in a humidified atmosphere with $5 \% \mathrm{CO}_{2}$ at $37{ }^{\circ} \mathrm{C}$. The clinical isolate $\mathrm{RVi} / \mathrm{Wuerzburg.DEU/47.11} \mathrm{(Wb-12,} \mathrm{genotype} \mathrm{2B)}$ was provided by Dr. Benedikt Weissbrich at the University of Wuerzburg, propagated on Vero76 cells (ATCC) and titered by the immunocolorimetric plaque assay [39].

For growth curve analysis, cells were seeded in 48-well culture plates at a density of $5^{\circ} 10^{4}$ cells per well and infected with Wb-12 at an MOI of 5 the following day. After adsorption for $2 \mathrm{~h}$ at $35{ }^{\circ} \mathrm{C}$, the viral inoculum was removed, the cells were washed three times with $1 x$ PBS and overlaid with $500 \mu \mathrm{l}$ fresh medium. Supernatants and cells were collected at the indicated time points. The amount of intracellular virus was determined by dissolving the cell pellet in $500 \mu \mathrm{l}$ fresh medium followed by three subsequent freeze-thaw cycles and centrifugation for $5 \mathrm{~min}$ at 400.g to remove debris. Virus titer of the supernatant was determined by immunocolorimetric plaque assay on Vero76 cells in duplicate.

\section{Microarray, hybridization and gene expression analysis}

For microarray analysis, $2^{\circ} 10^{6}$ endothelial cells of fetal and adult origin were infected with RV at an MOI of 10 and cells were lysed $36 \mathrm{~h}$ post infection with $600 \mu \mathrm{l}$ RLT buffer from the RNAeasy kit (Qiagen). RNA samples from three independently RV-infected or mock-infected cell cultures were used for each analysis. RNA-extraction, microarray chip hybridization and processing were performed by ATLAS Biolabs GmbH. DNA microarray analysis was carried out using the Affymetrix Human Genome U133 Plus 2.0 array (Affymetrix). The obtained data were further processed, checked for quality, and filtered using the Affymetrix Expression Console Software. Gene level analysis was further conducted with Affymetrix Transcriptome Analysis Console 2.0 software.

Analysis of enriched biological processes and Kyoto Encyclopedia of Genes and Genomes (KEGG) pathways of the differentially expressed genes after RV infection was carried out using the Cytoscape v 3.1.1 plugin ClueGO v2.1.1 [40]. Processes and pathways that possessed a p-value of $\leq 0.01$ were displayed. Term and group significance were calculated using a two-sided hypergeometric test and a Bonferroni correction of the p-value.

\section{Quantitative real-time PCR (qPCR)}

Total RNA from five independent infections of HUVEC and six independent infections of HSaVEC, was harvested $36 \mathrm{~h}$ post infection using the RNAeasy Mini Kit (Qiagen) according to manufacturer's instructions. In order to account for donor-to-donor variation, experiments with HUVEC were conducted using two different pools of cells (with each pool consisting of cells from three different donors); furthermore, experiments with HSaVEC were carried out on cells from two different donors. Approximately $6 \cdot 10^{5}$ cells were used per RNA isolation column and remaining DNA contaminants were removed by a 30 min digest with $20 \mathrm{U}$ of Turbo-DNase (Ambion). RNA was eluted twice with RNase-free water and the RNA concentration was determined using the NanoDrop 8000 (Thermo Scientific). For cDNA generation, $1 \mu \mathrm{g}$ of RNA was incubated for $1 \mathrm{~h}$ at $45^{\circ} \mathrm{C}$ with the following components: 1 unit RevertAid ${ }^{\text {Th }} \mathrm{H}$ minus reverse transcriptase, $5 \mu \mathrm{M}$ oligo $(\mathrm{dT}) 18$ primer, $1 \mathrm{x}$ reaction buffer, $1 \mathrm{mM}$ dNTP, and $20 \mathrm{U}$ RiboLock RNase inhibitor (Fermentas). The reaction was terminated by heating the mixture for $10 \mathrm{~min}$ at $70{ }^{\circ} \mathrm{C}$. Reactions were performed in a total volume of $25 \mu \mathrm{l}$, consisting of $1 \mathrm{x}$ SYBR $^{\circ}$ Green PCR Master Mix (Life Technology), forward and reverse primers (200 $\mathrm{nM}$ each) and $5 \mu \mathrm{l}$ of cDNA, dilutions ranging from 1:5 to 1:50. Cycling 
conditions were as follows: $95{ }^{\circ} \mathrm{C}$ for $15 \mathrm{~min}$, followed by 40 cycles of $95{ }^{\circ} \mathrm{C}$ for $15 \mathrm{~s}$ and $60{ }^{\circ} \mathrm{C}$ for $30 \mathrm{~s}$ using the LightCycler 480 system (Roche). Fluorescence readings were recorded at the last step. Melting curve analysis was performed after amplification to determine the presence of nonspecific amplification products. All primer pairs used in this study are listed in Additional file 5. Primers for the amplification of NOG were purchased from Biorad (Germany). Gene expression, normalized to $G A P D H$, was determined using the $\triangle \triangle \mathrm{Ct}$ mathematical model (Livak \& Schmittgen, 2001). The paired onetailed $t$-test was used to determine statistically significant differences in $\Delta C T$ values between non-infected and infected groups.

\section{Protein microarray and flow cytometry analysis}

For protein microarray analysis, $2 \cdot 10^{5}$ HUVEC and HSaVEC were infected with RV at an MOI of ten in two independent experiments. Supernatants were collected $48 \mathrm{hpi}$ and analyzed for the presence of chemokines using the Proteome Profiler Human Chemokine Array Kit (R\&D Systems, Minneapolis, MN, USA) according to the manufacturer's instructions. For flow cytometry analysis, $1^{\circ} 10^{5}$ HUVEC and HSaVEC were infected with RV at an MOI of 5 and detached with trypsin at various time points post infection. Following fixation with $2 \%$ paraformaldehyde for $20 \mathrm{~min}$, cells were permeabilized with $0.5 \%$ saponine and stained with a RV anti-capsid antibody (Meridian Life Science, USA) and a secondary goat anti-mouse APC (Biolegend, USA). Flow cytometry data were acquired on a FACSCalibur flow cytometer (BD Biosciences, USA) using Cell Quest Pro software (BD Biosciences).

\section{Additional files}

\section{Additional file 1: List of transcripts that were differentially regulated after RV infection. (PDF $241 \mathrm{~kb}$ )}

Additional file 2: Absolute chemokine expression following RV infection. (PDF $761 \mathrm{~kb}$ )

Additional file 3: Ranking of commonly up- and down-regulated genes in the endothelial cells following RV infection. (PDF $52 \mathrm{~kb}$ )

Additional file 4: Comparison of differentially regulated genes following RV infection in HUVEC, HSaVEC, HEF and Hs888Lu. (PDF $244 \mathrm{~kb}$ )

Additional file 5: List of primer sequences used for expression analysis of genes belonging to the GO term "sensory organ development" by qPCR. (PDF $7 \mathrm{~kb}$ )

\section{Abbreviations}

ADAMTS18: ADAM metallopeptidase with thrombospondin type 1 motif, 18; ALDH1A2: aldehyde dehydrogenase 1 family, member A2; CLN8: ceroidlipofuscinosis, neuronal 8; CPE: cytopathic effect; CRS: congenital rubella syndrome; DSCAM: down syndrome cell adhesion molecule; FGFR2: fibroblast growth factor receptor 2; FZD3: frizzled class receptor 3; GO: gene ontology; HSaVEC: human saphenous vein endothelial cells; HUVEC: human umbilical vein endothelial cells; JAG2: jagged 2; KEGG: Kyoto Encyclopedia of Genes and Genomes; MYO3A: myosin 3A; MYO7A: myosin 7A; NHS: Nance-Horan syndrome; NOG: noggin; NTRK3: neurotrophic tyrosine kinase, receptor, type 3; PDGFRA: platelet-derived growth factor receptor, alpha polypeptide; RDH10: retinol dehydrogenase 10; RV: rubella virus; SLC25A27: solute carrier family 25, member 27; TNPO1: transportin 1; TRPM1: transient receptor potential cation channel, subfamily M, member 1; TSPAN12: tetraspanin 12.

\section{Competing interests}

All authors declare that they have no competing interests.

\section{Authors' contributions}

HG was responsible for design, conduct, study completion and statistical analysis. $\mathrm{HG}$ and $\mathrm{AL}$ were responsible for writing the manuscript. MB was responsible for the viral growth curve, detection of viral protein by flow cytometry, infection for microarray analysis and statistical analysis. JN and HG carried out the chemokine profiler and quantitative GPCR. AL and NF participated in the statistical analysis. PR carried out the microscopic analysis. CC and LP participated in the study design and work with the primary endothelial cells. AM was the principal investigator and was responsible for research design and coordination. All authors read and approved the manuscript.

\section{Acknowledgment}

We would like to thank Dr. Benedikt Weissbrich for providing the Wb-12 strain used in this work. This research was supported by the Robert Koch Institute.

\section{Author details}

'Division 12, "Measles, Mumps, Rubella, and Viruses Affecting Immunocompromised Patients", Robert Koch Institute, 13353 Berlin, Germany. ${ }^{2}$ Institute of Molecular Life Sciences, University of Zurich, 8057 Zurich, Switzerland. "Unit "Diagnostics and Pathogen Characterisation", Bundesinstitut für Risikobewertung, 12277 Berlin, Germany. ${ }^{4}$ Institut für Virologie, Universität Leipzig, Johannisallee 30, 04103 Leipzig, Germany. ${ }^{5}$ Division of Viral Diseases, Centers for Disease Control and Prevention, 1600 Clifton Rd, Atlanta, GA 30333, USA.

Received: 30 October 2015 Accepted: 25 January 2016

Published online: 02 February 2016

\section{References}

1. Hobman T, Chantler J. In: Knipe DM, Howley PM, editors. Fields Virology Fifth ed, vol One. Philadelphia: Lippincott Williams \& Wilkins; 2001. p. 963-90.

2. Saraswathy TS, Rozainanee MZ, Asshikin RN, Zainah S. Congenital rubella syndrome: a review of laboratory data from 2002 to 2011. Southeast Asian J Trop Med Public Health. 2013;44:429-35.

3. Webster WS. Teratogen update: congenital rubella. Teratology. 1998; 58:13-23.

4. Plotkin SA. Routes of fetal infection and mechanisms of fetal damage. Am J Dis Child. 1975;129:444-9.

5. Kistler GS. Cytoplasmic tubuloreticular complexes and nuclear bodies in cells of rubella-infected human embryos and fetuses (author's transl). Beitr Pathol. 1975;155:101-38.

6. Menser MA, Reye RD. The pathology of congenital rubella: a review written by request. Pathology. 1974;6:215-22.

7. Tondury G, Smith DW. Fetal rubella pathology. J Pediatr. 1966;68:867-79.

8. Gregg NM. Congenital cataract following German measles in the mother. Trans Ophthalmol Soc Aust. 1941;3:35-46.

9. Rowe RD. Cardiovascular disease in the rubella syndrome. Cardiovasc Clin. 1973;5:61-80.

10. Banatvala JE, Brown DW. Rubella. Lancet. 2004;363:1127-37.

11. Reef SE, Strebel P, Dabbagh A, Gacic-Dobo M, Cochi S. Progress toward control of rubella and prevention of congenital rubella syndromeworldwide, 2009. J Infect Dis. 2011;204 Suppl 1:S24-7.

12. Lee JY, Bowden DS. Rubella virus replication and links to teratogenicity. Clin Microbiol Rev. 2000;13:571-87.

13. Knipe DM, Howley PM. Fields Virology. Philadelphia: Lippincott Williams \& Wilkins; 2007.

14. Adamo MP, Zapata M, Frey TK. Analysis of gene expression in fetal and adult cells infected with rubella virus. Virology. 2008;370:1-11. 
15. Mo XY, Ma W, Zhang $Y$, Zhao $H$, Deng $Y$, Yuan $W$, et al. Microarray analyses of differentially expressed human genes and biological processes in ECV304 cells infected with rubella virus. J Med Virol. 2007;79:1783-91.

16. Petruzziello R, Orsi N, Macchia S, Rieti S, Frey TK, Mastromarino P. Pathway of rubella virus infectious entry into Vero cells. J Gen Virol. 1996;77:303-8.

17. Hemphill ML, Forng RY, Abernathy ES, Frey TK. Time Course of Virus-Specific Macromolecular Synthesis During Rubella Virus Infection in Vero Cells. Virology. 1988;162:65-75.

18. Frey TK. Molecular biology of rubella virus. Adv Virus Res. 1994;44:69-160.

19. Perelygina $L$, Zheng $Q$, Metcalfe $M$, Icenogle J. Persistent infection of human fetal endothelial cells with rubella virus. PLoS ONE. 2013;8:e73014.

20. Bardeletti G, Tektoff J, Gautheron D. Rubella virus maturation and production in two host cell systems. Intervirology. 1979;11:97-103.

21. Sedwick WD, Sokol F. Nucleic acid of rubella virus and its replication in hamster kidney cells. J Virol. 1970;5:478-89.

22. Niu J, Kolattukudy PE. Role of MCP-1 in cardiovascular disease: molecular mechanisms and clinical implications. Clin Sci (Lond). 2009;117:95-109.

23. van den Borne P, Quax PH, Hoefer IE, Pasterkamp G. The multifaceted functions of CXCL10 in cardiovascular disease. Biomed Res Int. 2014; 2014:893106.

24. Aukrust $\mathrm{P}$, Halvorsen $\mathrm{B}$, Yndestad $\mathrm{A}$, Ueland $\mathrm{T}$, Oie $\mathrm{E}$, Otterdal $\mathrm{K}$, et al. Chemokines and cardiovascular risk. Arterioscler Thromb Vasc Biol. 2008; 28:1909-19.

25. Hannan NJ, Jones RL, White CA, Salamonsen LA. The chemokines, CX3CL1, CCL14, and CCL4, promote human trophoblast migration at the fetomaternal interface. Biol Reprod. 2006;74:896-904.

26. Chan G, Bivins-Smith ER, Smith MS, Smith PM, Yurochko AD. Transcriptome analysis reveals human cytomegalovirus reprograms monocyte differentiation toward an M1 macrophage. J Immunol. 2008;181:698-711.

27. Mahajnah M, Zelnik N. Phenotypic heterogeneity in consanguineous patients with a common CLN8 mutation. Pediatr Neurol. 2012;47:303-5.

28. Brooks SP, Coccia M, Tang HR, Kanuga N, Machesky LM, Bailly M, et al. The Nance-Horan syndrome protein encodes a functional WAVE homology domain (WHD) and is important for co-ordinating actin remodelling and maintaining cell morphology. Hum Mol Genet. 2010;19:2421-32.

29. Qu Y, Huang Y, Feng J, Alvarez-Bolado G, Grove EA, Yang Y, et al. Genetic evidence that Celsr3 and Celsr2, together with Fzd3, regulate forebrain wiring in a Vangl-independent manner. Proc Natl Acad Sci U S A. 2014;111: E2996-3004

30. Kiernan AE, Cordes R, Kopan R, Gossler A, Gridley T. The Notch ligands DLL1 and JAG2 act synergistically to regulate hair cell development in the mammalian inner ear. Development. 2005;132:4353-62.

31. Lanford PJ, Lan Y, Jiang R, Lindsell C, Weinmaster G, Gridley T, et al. Notch signalling pathway mediates hair cell development in mammalian cochlea. Nat Genet. 1999:21:289-92.

32. Chang W, Nunes FD, De Jesus-Escobar JM, Harland R, Wu DK. Ectopic noggin blocks sensory and nonsensory organ morphogenesis in the chicken inner ear. Dev Biol. 1999;216:369-81.

33. Gerlach LM, Hutson MR, Germiller JA, Nguyen-Luu D, Victor JC, Barald KF. Addition of the BMP4 antagonist, noggin, disrupts avian inner ear development. Development. 2000;127:45-54.

34. McMahon JA, Takada S, Zimmerman LB, Fan CM, Harland RM, McMahon AP. Noggin-mediated antagonism of BMP signaling is required for growth and patterning of the neural tube and somite. Genes Dev. 1998;12:1438-52.

35. Smorodchenko A, Rupprecht A, Fuchs J, Gross J, Pohl EE. Role of mitochondrial uncoupling protein 4 in rat inner ear. Mol Cell Neurosci. 2011; 47:244-53.

36. Merrill AE, Sarukhanov A, Krejci P, Idoni B, Camacho N, Estrada KD, et al. Bent bone dysplasia-FGFR2 type, a distinct skeletal disorder, has deficient canonical FGF signaling. Am J Hum Genet. 2012;90:550-7.

37. Jaijo T, Aller E, Beneyto M, Najera C, Graziano C, Turchetti D, et al. MYO7A mutation screening in Usher syndrome type I patients from diverse origins. J Med Genet. 2007;44:e71.

38. Eudy JD, Sumegi J. Molecular genetics of Usher syndrome. Cell Mol Life Sci. 1999;56:258-67.

39. Chen MH, Zhu Z, Zhang Y, Favors S, Xu WB, Featherstone DA, et al. An indirect immunocolorimetric assay to detect rubella virus infected cells. J Virol Methods. 2007;146:414-8.

40. Bindea G, Mlecnik B, Hackl H, Charoentong P, Tosolini M, Kirilovsky A, et al. ClueGO: a Cytoscape plug-in to decipher functionally grouped gene ontology and pathway annotation networks. Bioinformatics. 2009;25:1091-3.

\section{Submit your next manuscript to BioMed Central and we will help you at every step:}

- We accept pre-submission inquiries

- Our selector tool helps you to find the most relevant journal

- We provide round the clock customer support

- Convenient online submission

- Thorough peer review

- Inclusion in PubMed and all major indexing services

- Maximum visibility for your research

Submit your manuscript at www.biomedcentral.com/submit
Biomed Central 